\title{
STORYTELLING DIGITAL EN COMUNICACIÓN CORPORATIVA: COMPARATIVA ENTRE IBEX 35 Y DOW JONES
}

\section{Digital storytelling in corporate communication: A comparison between IBEX 35 and the Dow Jones}

Luis Mañas-Viniegra

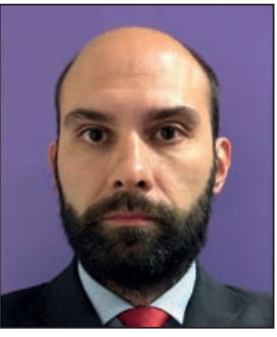

Luis Mañas-Viniegra es doctor en Comunicación Audiovisual y Publicidad, profesor asociado en las Universidades Complutense de Madrid (UCM) y Rey Juan Carlos y miembro del Grupo de Investigación Gestión de las Marcas y Procesos de Comunicación (UCM). Profesionalmente es marketing manager en Jetcommunication.net, entre cuyos clientes se encuentran empresas cotizadas en bolsas europeas. Anteriormente ha sido director de agencia de publicidad.

http://orcid.org/0000-0001-9129-5673

Universidad Complutense de Madrid Facultad de Ciencias de la Información, Departamento de Comunicación Audiovisual y Publicidad Avda. Complutense, s/n. 28040 Madrid, España Imanas@ucm.es

\section{Resumen}

Las webs corporativas de las marcas se enfrentan al reto de incorporar una narrativa de storytelling que fomente los vínculos emocionales con sus públicos. Este trabajo establece cuatro categorías de implantación en las empresas del IBEX 35 y pone de manifiesto su demora frente a las empresas que cotizan en el Dow Jones. Los casos de estudio extraídos permiten establecer recomendaciones que mejoren esta situación.

\section{Palabras clave}

Storytelling; Marca corporativa; Interactividad; Parallax; Storytelling digital; Empresas; Sitios web.

\section{Abstract}

Corporate websites have been challenged to incorporate storytelling narratives that favor emotional bonds with their stakeholders. This paper establishes four categories of implementation in the companies of IBEX 35 and reveals its delay of companies listed in the Dow Jones index. The case studies presented allow us to make recommendations in order to improve the situation.

\section{Keywords}

Storytelling; Corporate brand; Interactivity; Parallax; Digital storytelling; Companies; Web sites.

Mañas-Viniegra, Luis (2017). "Storytelling digital en comunicación corporativa: comparativa entre IBEX 35 y Dow Jones". El profesional de la información, v. 26, n. 6, pp. 1127-1138.

\section{https://doi.org/10.3145/epi.2017.nov.12}

\section{Introducción}

La aparición de la web 2.0 (O’Reilly, 2005) inició la interactividad y participación de los públicos en los contenidos digitales de las marcas, inmersas aún en su transición desde la marca producto hacia la marca corporativa (Benavides, 2013), en la que su identidad como empresa contribuye a generar reputación. Por otra parte, las empresas también son vistas como el principal motivo de los conflictos sociales, ambientales y económicos, y registran niveles mínimos de legitimidad (Porter; Kramer, 2011). Y ello a pesar de la comunicación de sus acciones de responsabilidad social, que han hecho de forma unidireccional y no interactiva (Pineda-Martínez, 2013, pp. 189-203), sin obtener credibilidad. Ante esta situación es prioritario para las marcas fomentar los vínculos emocionales con sus públicos hasta convertirse en una lovemark (Roberts, 2005).

La web corporativa es el primer soporte digital a partir del cual proyectar esa identidad de empresa, por lo que 
"es necesario recoger todos los elementos visuales relacionados con la imagen de marca institucional" (Pérez-Montoro, 2014, p. 192).

En la información corporativa de las empresas del IBEX 35, el $97,4 \%$ incorpora su historia y el $100 \%$ los informes financieros (Tapia-Frade; Caerlos-Mateo, 2014). Las grandes empresas españolas muestran de manera digital su identidad y sus resultados, a pesar de que la Fundación Compromiso y Transparencia (2013) suspenda al $100 \%$ de las compañías del IBEX 35 en transparencia a partir de la información suministrada en sus webs.

\section{Para las marcas es prioritario el fomen- to de los vínculos emocionales con sus públicos}

Las empresas han centrado sus esfuerzos en crear salas de prensa virtuales en sus webs. Durántez-Stolle (2017) concluye que la interactividad más frecuente en empresas del IBEX 35 es la relacionada con la recuperación de información de interés ( $50,8 \%$ de los casos), mientras que el diálogo a través de medios sociales y el uso de medios para favorecer la viralidad de la información publicada es bajo (31,9\% y $31,7 \%$, respectivamente). Continúa existiendo una

"emisión unilateral de información" (Durántez-Stolle, 2017, p. 234).

Una dificultad en la administración de las webs corporativas es la saturación de contenidos, idónea para quien busca información muy específica, pero irrelevante para la mayoría de personas, que desean una aproximación más general. Con estos condicionantes las salas de prensa virtual son un medio poco efectivo para obtener esa vinculación emocional con los públicos.

El storytelling es una alternativa a esta problemática en los contenidos de webs corporativas. El $57 \%$ de los directores de comunicación consideran que será la disciplina más importante en los próximos años (González-Ortín, 2014, p. 16).

Herrero-Curiel (2005, p. 2) define el storytelling digital como

"una moderna expresión para el antiguo arte de contar historias",

que combina la narrativa con el contenido y el propósito de transmitir un mensaje con finalidad emocional. La tecnología permite además

"una mayor inmersión en la estructura narrativa" (Nakevska et al., 2017, p. 7).

Salmon (2008) considera que en la actual "era del relato" las historias contadas deben resultar creíbles e incide en su carácter emocional. Estas historias

"forman parte intrínseca de nuestra naturaleza como seres sociales" (Pereira-Lima, 2014, p. 122),

de modo que los públicos se identifican

"con unos contenidos que hacen suyos por propio convencimiento" (Martín-González, 2009, p. 9).
Actualmente las marcas construyen su discurso sobre los elementos que fundamentan la propia vida de sus públicos:

"sus relaciones, sus aspiraciones, sus éxitos, sus certezas, sus incertidumbres, sus anhelos y sus vivencias" (Martín-Requero; Alvarado-López, 2007, p. 11).

El storytelling resulta útil para construir la marca corporativa y debe incorporar sus valores (Adecec, 2015, p. 6; Costa-Sánchez, 2014, p. 166), definiendo la propia marca su personalidad. Fog, Budtz y Yakaboylu (2005) identifican además cuatro elementos del storytelling:

- mensaje central positivo;

- problema a resolver que construye la historia y la aleja de lo predecible y lo aburrido;

- argumento y personajes, donde la marca se muestra como el héroe de la historia;

- valores e identidad de una marca, que a través de las historias que comparten con sus públicos son comprensibles, comunican emociones y refuerzan los vínculos.

Las historias en el entorno digital se deben contar con un final abierto, enlazadas, participativas, exploratorias e impredecibles, siendo las historias en la web un contenido parcial y no total, destinado

"a ser reutilizado en múltiples formas y lugares" (Alexander; Levine, 2008, p. 42).

Fog, Budtz y Yakaboylu (2005) consideran que cualquier empresa puede generar historias a partir de:

- su fundador;

- éxitos y crisis transcurridas;

- el liderazgo;

- historias de los empleados;

- la credibilidad de los líderes de opinión;

- los clientes;

- los propios productos.

La narrativa es el elemento esencial del storytelling, a pesar de la aportación interactiva del ámbito digital (Lugmanyr et al., 2016, pp. 5-6)

La marca ya no tiene el control sobre la historia contada en el escenario digital. Son los públicos quienes la aceptan o rechazan, interpretan, matizan e implementan. Ya no es posible imponer un discurso oficial por parte de la empresa y esa implicación de los púbicos es la que favorece los vínculos emocionales gracias al entretenimiento que genera y a la experiencia que se comparte.

Tabla 1. Diferencias entre web corporativa y storytelling digital corporativo.

\begin{tabular}{|l|l|}
\hline \multicolumn{1}{|c|}{ Web corporativa } & Storytelling digital corporativo \\
\hline Marca de producto o corporativa & Marca corporativa \\
\hline Contenido y tecnología & Narrativa, contenido y tecnología \\
\hline Secciones temáticas & Historias, experiencias y valores \\
\hline Información unidireccional & Información interactiva \\
\hline Interés decreciente del contenido & Interés continuo del contenido \\
\hline Mensaje informativo y persuasivo & Mensaje creíble y emocional \\
\hline Leer & Participar y compartir \\
\hline
\end{tabular}




\section{Metodología}

\subsection{Método, objetivos e hipótesis}

Este trabajo estudia la presencia de storytelling en las webs corporativas de las empresas del IBEX 35 y su grado de implantación con respecto a las empresas cotizadas en el Dow Jones Industrial Average.

La hipótesis de partida es que las grandes compañías aún no han adoptado en sus webs las ventajas que proporciona el storytelling en su relación con los públicos.

Los objetivos son:

- establecer los niveles de aplicación de storytelling en las webs corporativas de empresas del IBEX 35;

- determinar su presencia;

- identificar casos destacados en el desarrollo de narrativas de este tipo;

- recomendar tecnologías que contribuyan a construir el storytelling digital.

La metodología aplicada es un análisis de contenido que parte de un censo compuesto por las 35 webs corporativas de las empresas del IBEX 35 y las 30 del Dow Jones. Como paso previo se ha realizado una observación exploratoria de las webs de los 100 mayores anunciantes en España según datos de Infoadex (Sánchez-Revilla, 2017) para identificar casos de éxito que permitiesen extraer las categorías a considerar, dada la ausencia bibliográfica al respecto.

Se ha realizado una comparativa a partir de la observación (Quivy; Van-Campenhoudt, 2008, p. 164) durante enero y febrero de 2017 de los cuatro niveles que configuran el storytelling digital corporativo. En la ficha de análisis creada ad hoc se han valorado las categorías escogidas en función de la presencia o ausencia de los requisitos mínimos establecidos en cada nivel.

Debido a la escasa implantación identificada de storytelling digital corporativo, se han agrupado las empresas en los 6 sectores en los que se estructura la Bolsa de Madrid. Se han reajustado los 19 súper sectores en los que se estructura el Dow Jones conforme a los 6 sectores españoles. Posteriormente se especifica qué empresas son casos de éxito en storytelling. El desglose de las empresas por sector se muestra en la tabla 2.

\subsection{Propuesta de categorías}

Se han establecido cuatro categorías de presencia de storytelling en una web corporativa:

\section{Nivel 1}

La máxima implementación se encuentra en una página de inicio o index construida desde el storytelling, que requerirá un reajuste de las historias contadas en función de la actualidad y no sólo de la estrategia de la organización. Cuando hablamos de storytelling, la página de inicio narra los valores, acontecimientos, la identidad... de la organización de forma progresiva y coordinada y no como una mera sucesión de datos relevantes de cada sección de la web. El criterio para valorar el cumplimiento de este máximo nivel es que esta página esté basada en una forma de storytelling.
La herramienta utilizada habitualmente para dinamizar el avance de la narrativa es una animación parallax, usada también por Apple en su storytelling digital e inspirada en la cámara multiplano utilizada por Disney a partir del invento de Frederic E. Ives (Uspto, 1903). Parallax se basa en una animación que hace que el fondo y el contenido, que suele combinar foto, vídeo y texto, se muevan a diferentes velocidades a medida que se desciende por el contenido de la página web mediante desplazamiento vertical o scroll. Ocupa toda la pantalla y contribuye a animar la transición entre los elementos de la historia, que podrían resultar independientes si no fuese gracias a este efecto. Aporta continuidad y dinamismo. Parallax es divertido, interactivo, crea una sensación de tridimensionalidad y rompe el tópico de que la información relevante en una web se encuentra siempre en la parte superior de la misma, porque anima a seguir la historia al completo (UXPin, 2015). Los vídeos interactivos son además un complemento a parallax en el soporte a la narrativa.

Tabla 2. Empresas cotizadas agrupadas por sectores

\begin{tabular}{|c|c|c|}
\hline Sectores & $\begin{array}{c}\text { Empresas } \\
\text { IBEX } 35\end{array}$ & $\begin{array}{l}\text { Empresas } \\
\text { Dow Jones }\end{array}$ \\
\hline 1. Petróleo y energía & $\begin{array}{l}\text { 1. Enagas } \\
\text { 2. Endesa } \\
\text { 3. Gas Natural } \\
\text { 4. Iberdrola } \\
\text { 5. REE } \\
\text { 6. Repsol }\end{array}$ & $\begin{array}{l}\text { 1. Chevron } \\
\text { 2. Exxon Mobil } \\
\text { 3. General Electric }\end{array}$ \\
\hline $\begin{array}{l}\text { 2. Materiales básicos, } \\
\text { industria y } \\
\text { construcción }\end{array}$ & $\begin{array}{l}\text { 1. Acciona } \\
\text { 2. Acerinox } \\
\text { 3. ACS } \\
\text { 4. Arcelormitttal } \\
\text { 5. Ferrovial } \\
\text { 6. Gamesa } \\
\text { 7. Técnicas Reunidas }\end{array}$ & $\begin{array}{l}\text { 1. } 3 M \\
\text { 2. Boeing Co } \\
\text { 3. Caterpillar } \\
\text { 4. Du Pont (EI) de NMR } \\
\text { 5. United Technologies }\end{array}$ \\
\hline 3. Bienes de consumo & $\begin{array}{l}\text { 1. Grifols } \\
\text { 2. Inditex } \\
\text { 3. Viscofan }\end{array}$ & $\begin{array}{l}\text { 1. Coca-Cola } \\
\text { 2. Johnson \& Johnson } \\
\text { 3. Merck and Co. New } \\
\text { 4. Nike } \\
\text { 5. Pfizer } \\
\text { 6. Procter \& Gamble }\end{array}$ \\
\hline $\begin{array}{l}\text { 4. Servicios de } \\
\text { consumo }\end{array}$ & $\begin{array}{l}\text { 1. Abertis } \\
\text { 2. AENA } \\
\text { 3. DIA } \\
\text { 4. IAG } \\
\text { 5. Mediaset } \\
\text { 6. Meliá Hotels Intl }\end{array}$ & $\begin{array}{l}\text { 1. Home Depot } \\
\text { 2. Mc Donald's Corp } \\
\text { 3. Wal-Mart Stores } \\
\text { 4. Walt Disney Co }\end{array}$ \\
\hline $\begin{array}{l}\text { 5. Servicios financie- } \\
\text { ros e inmobiliarios }\end{array}$ & $\begin{array}{l}\text { 1. Bankia } \\
\text { 2. Bankinter } \\
\text { 3. BBVA } \\
\text { 4. Caixabank } \\
\text { 5. Mapfre } \\
\text { 6. Merlin Properties } \\
\text { 7. Popular } \\
\text { 8. Sabadell } \\
\text { 9. Santander }\end{array}$ & $\begin{array}{l}\text { 1. American Express } \\
\text { 2. Goldman Sachs } \\
\text { 3. JP Morgan Chase } \\
\text { 4. Travelers Cies } \\
\text { 5. United Health Group } \\
\text { 6. VISA }\end{array}$ \\
\hline $\begin{array}{l}\text { 6. Tecnología y } \\
\text { telecomunicaciones }\end{array}$ & $\begin{array}{l}\text { 1. Amadeus } \\
\text { 2. Cellnex Telecom } \\
\text { 3. Indra } \\
\text { 4. Telefónica }\end{array}$ & $\begin{array}{l}\text { 1. Apple } \\
\text { 2. Cisco Systems } \\
\text { 3. IBM } \\
\text { 4. Intel Corp } \\
\text { 5. Microsoft } \\
\text { 6. Verizon Comms }\end{array}$ \\
\hline $\begin{array}{l}\text { Total empresas } \\
\text { cotizadas }\end{array}$ & 35 & 30 \\
\hline
\end{tabular}




\section{Nivel 2}

Utilización de storytelling en algún lugar de la web para contar historias o stories sobre el producto o los públicos de interés. En este nivel 2, la animación parallax también es una herramienta idónea. Los vídeos interactivos y 360 o son tecnologías indicadas.

\section{El storytelling digital se puede construir con tecnologías como animación para- Ilax, infografías, líneas de tiempo inte- ractivas...}

\section{Nivel 3}

Presencia de storytelling en la comunicación de los resultados financieros. La herramienta que mejor se adapta a esta narrativa son las infografías sobre el modelo de negocio y los propios resultados, con el fin de que la información sea sintetizada, comprensible para todos los públicos, interactiva, apta para ser compartida en redes sociales y susceptible de ser comentada. Aunque en el estudio exploratorio también se detectaron infografías sobre procesos, valores u otros relatos, éstas existían únicamente cuando con carácter previo estaban presentes las infografías sobre modelos de negocio y resultados financieros.

Cairo (2008, pp. 28-29) identifica dos funciones en las infografías: estética y analítica. La primera es la indicada para las noticias periodísticas, mientras que la segunda incrementa la capacidad de comprensión del lector, puesto que se establecen relaciones entre los hechos mostrados visualmente. Ambas funciones se cumplen en todos los casos identificados.

Sin embargo, no podemos obviar otras dos funciones esenciales para alcanzar el vínculo emocional con el público que diferencia una infografía periodística de otra orientada hacia la marca corporativa (figura 2), como son la interacción y la capacidad de ser compartida. Así lo reconocen Túñez-López y Nogueira (2017, pp. 154-155) al señalar como principales carencias de las infografías periodísticas el contenido y la animación. La infografía no puede agotarse en una síntesis visual (Colle, 2004; Valero-Sancho, 2008), por lo que debe proporcionar la posibilidad de ampliar esa información mediante un enlace.

Las marcas corporativas compiten en el mercado de la reputación (Johndrow, 2010) y deben considerar que sus infografías deben reflejar cómo han logrado que el beneficio aumente en un contexto socialmente responsable, de transparencia y buen gobierno. La infografía no se reduce a un mero gráfico que muestre la evolución de la cotización de la acción, por muy interactivo que sea. Se han encontrado gráficos en la web, las presentaciones asociadas a las Juntas Generales de Accionistas o Memorias Anuales, que son parciales, aislados, sin una narrativa y, por tanto, sin storytelling. En último lugar hay que considerar que un PDF no facilita la participación e interacción, aunque se haya estimado el contenido.

\section{Nivel 4}

Representa el menor grado de adaptación al storytelling, que se encuentra presente en la transformación de largos textos o vídeos sobre la historia de la empresa en un relato sobre su historia, acontecimientos clave y valores. La herramienta idónea es la línea de tiempo interactiva o timeline con hipermedialidad, en la que una breve información se amplía si hay interés.

\section{Resultados y discusión}

\section{Análisis exploratorio}

El análisis de las webs de los 100 mayores anunciantes en España sólo permitió encontrar un caso de éxito, el de Heineken, que aun no disponiendo de storytelling en su página de inicio sí está presente en toda la web con los requisitos de nivel 2 (stories). El avance de la narrativa se soporta sobre una animación parallax.

La directora de comunicación de Heineken afirmó en la presentación de la web que "para llegar a los usuarios en internet, hemos de ser capaces de generar contenidos de calidad que vayan más allá del enfoque puramente promocional" (Marketingdirecto.com, 2015).

A pesar de ello, el storytelling se dirige al producto y no a la marca corporativa.

El nivel 4 está presente al narrar la historia de la empresa, aunque no cuenta con timeline ni elementos interactivos. En cambio, el nivel 3 no, a pesar de que la narrativa y las

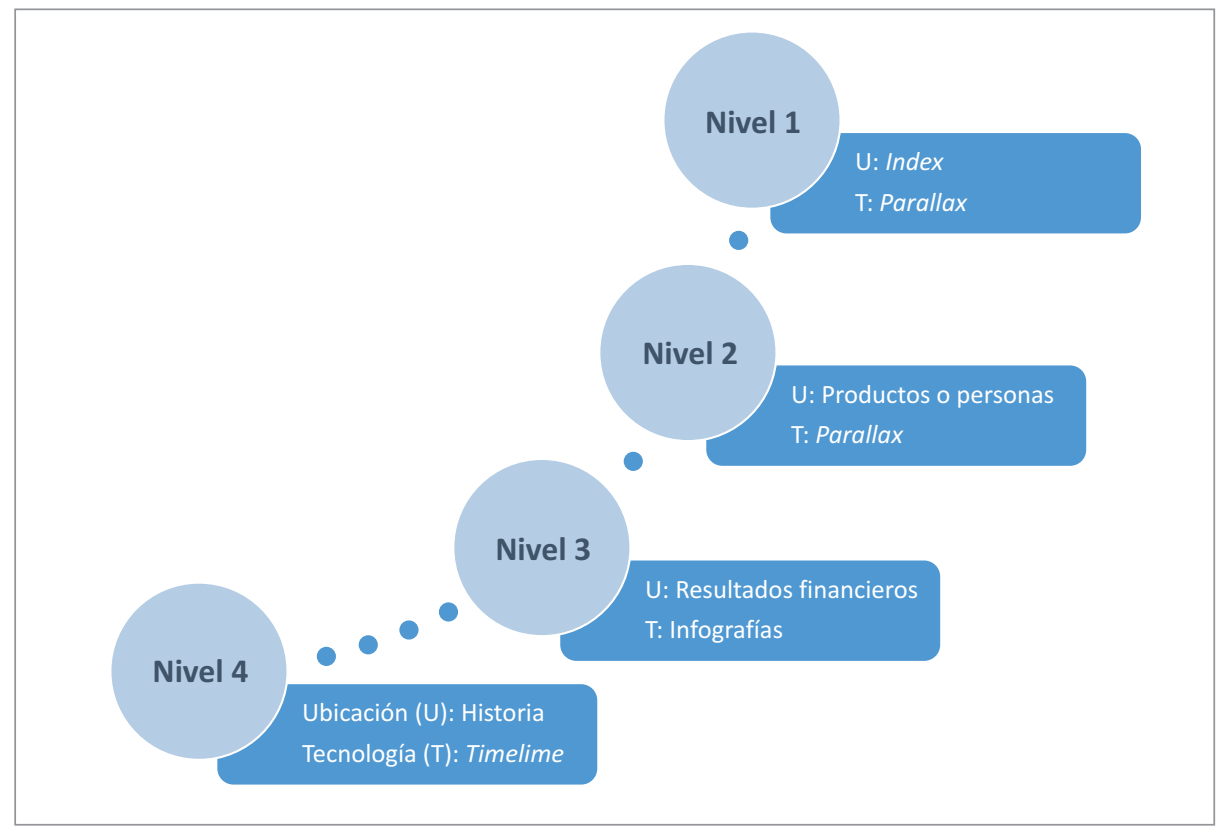

Figura 1. Niveles de presencia de storytelling digital corporativo 


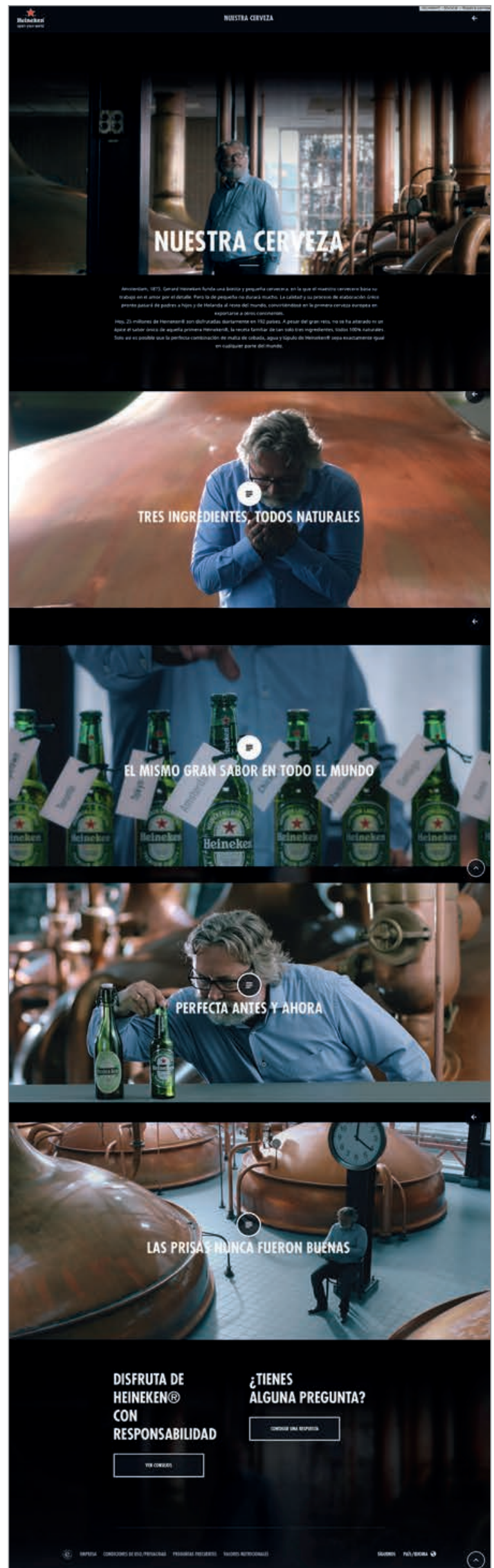

Figura 2. Storytelling de nivel 2 en la web de Heineken. http://goo.gl/bQqfuA
Diana Zreik

+2 Seguir

@DianaZreik

Peleas de perros patrocinadas por la cerveza@Heineken lamentable. Bebe San Miguel $100 \%$ española.

pic.twitter.com/UXVia7yYWz

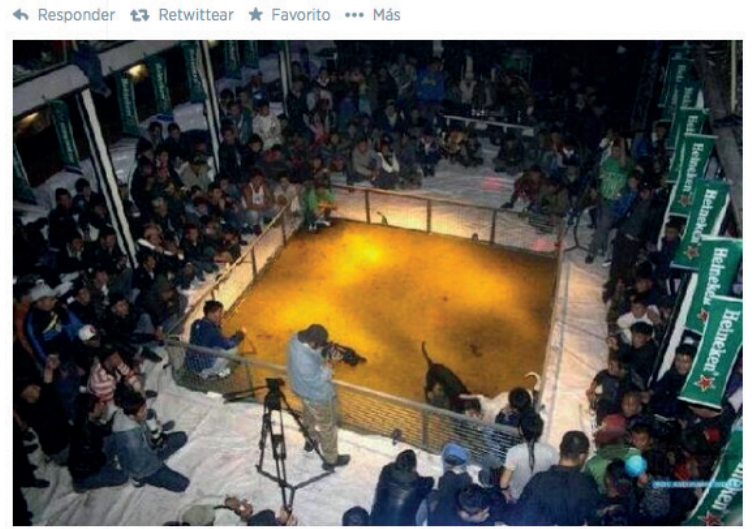

$\begin{array}{llll}\text { Retwets } & \text { favortos } & 2 & 2\end{array}$

12:23 - 10 de jun. de 2013

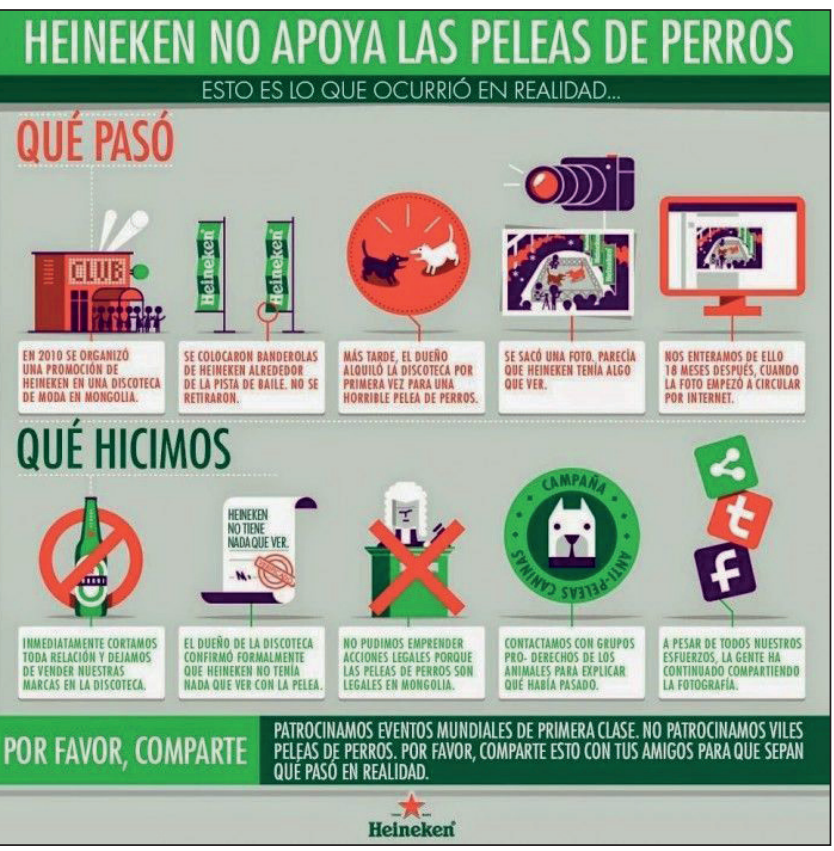

Figura 3. Información falsa sobre Heineken e infografía creada por la marca para combatirla.

$h t t p: / / g o o . g l / f P G H 7 P$

http://goo.gl/YCBHes

infografías son muy empleadas por la marca en su comunicación en redes sociales, tanto para productos como para información financiera o responsabilidad social corporativa. Incluso se han empleado para la comunicación de crisis, como en el ejemplo de respuesta que dio la empresa para combatir una información falsa que emerge cada cierto tiempo relacionada con unas supuestas peleas de perros que los públicos creen patrocinadas por Heineken (figura 3).

Continúan predominando casos como el de El Corte Inglés, segundo anunciante por volumen de inversión publicitaria. 
Esta empresa comunica su historia en modo texto con una sucesión interminable de fechas y acontecimientos a través de 1.619 palabras, dispone de una única infografía sobre su impacto socioeconómico sin narrativa y el index de su página corporativa se reduce a una sucesión de noticias ordenadas de forma cronológica.

\section{Las marcas construyen su discurso sobre} los elementos que fundamentan la propia vida de sus públicos

\section{Análisis del IBEX 35}

El análisis refleja una escasa implantación del storytelling en sus diferentes niveles. Ninguna empresa lo incluye en su index y sólo una, BBVA, lo hace en formato stories desde la óptica de sus productos. Hay que considerar que BBVA es la empresa española pionera en el periodismo de marca (Cole; Greer, 2013; Bull, 2013; Barciela, 2013), creando portales informativos que van más allá de una mera sala de prensa virtual y que compiten con los propios medios de comunicación online en cuanto a oferta informativa e incluso audiencias. Por ello debe entenderse como una evolución natural dentro de su estrategia de contenidos como medio para relacionarse con sus públicos, que además genera reputación.

\section{Ninguna empresa del IBEX 35 alcanza el máximo grado de storytelling digital cor- porativo y sólo una lo aplica orientado hacia el producto}

Se obtienen discretos resultados en los niveles 4 (34,29\% cumplen los requisitos mínimos) y 3 (37,14\% de las empresas). En estas dos categorías, los datos por sectores son dispares, puesto que los mejores resultados se encuentran en petróleo y energía ( $60 \%$ de los casos) y en servicios financieros e inmobiliarios $(44,44 \%)$, mientras que en su aplicación a la historia de la entidad son bienes de consumo $(66,67 \%)$ y servicios de consumo (42,87\%). Petróleo y energía por un lado, y servicios financieros e inmobiliarios por otro, que habían obtenido los dos mejores resultados en el nivel 3 , obtienen los dos peores en el nivel 1 , con un $20 \%$ y un $11,11 \%$ de los casos respectivamente. Los datos muestran una implementación escasa y desigual del storytelling en la comunicación digital de las empresas.

Tabla 3. Presencia de categorías de storytelling por sectores del IBEX 35.

\begin{tabular}{|c|c|c|c|c|}
\hline Categorías & Sectores & No empresas & $\%$ & $\%$ \\
\hline \multirow{6}{*}{ Nivel 1} & 1. Petróleo y energía & 0 & 0,00 & \multirow{6}{*}{0,00} \\
\hline & 2. Mats. básicos, industria y construcción & 0 & 0,00 & \\
\hline & 3. Bienes de consumo & 0 & 0,00 & \\
\hline & 4. Servicios de consumo & 0 & 0,00 & \\
\hline & 5. Servicios financieros e inmobiliarios & 0 & 0,00 & \\
\hline & 6. Tecnología y telecomunicaciones & 0 & 0,00 & \\
\hline \multirow{6}{*}{ Nivel 2} & 1. Petróleo y energía & 0 & 0,00 & \multirow{6}{*}{2,86} \\
\hline & 2. Mats. básicos, industria y construcción & 0 & 0,00 & \\
\hline & 3. Bienes de consumo & 0 & 0,00 & \\
\hline & 4. Servicios de consumo & 0 & 0,00 & \\
\hline & 5. Servicios financieros e inmobiliarios & 1 & 11,11 & \\
\hline & 6. Tecnología y telecomunicaciones & 0 & 0,00 & \\
\hline \multirow{6}{*}{ Nivel 3} & 1. Petróleo y energía & 4 & 66,67 & \multirow{6}{*}{40,00} \\
\hline & 2. Mats. básicos, industria y construcción & 2 & 28,67 & \\
\hline & 3. Bienes de consumo & 1 & 33,33 & \\
\hline & 4. Servicios de consumo & 2 & 28,57 & \\
\hline & 5. Servicios financieros e inmobiliarios & 4 & 44,44 & \\
\hline & 6. Tecnología y telecomunicaciones & 1 & 20,00 & \\
\hline \multirow{6}{*}{ Nivel 4} & 1. Petróleo y energía & 1 & 16,67 & \multirow{6}{*}{34,29} \\
\hline & 2. Mats. básicos, industria y construcción & 3 & 42,86 & \\
\hline & 3. Bienes de consumo & 2 & 66,67 & \\
\hline & 4. Servicios de consumo & 3 & 42,87 & \\
\hline & 5. Servicios financieros e inmobiliarios & 1 & 11,11 & \\
\hline & 6. Tecnología y telecomunicaciones & 2 & 40,00 & \\
\hline
\end{tabular}




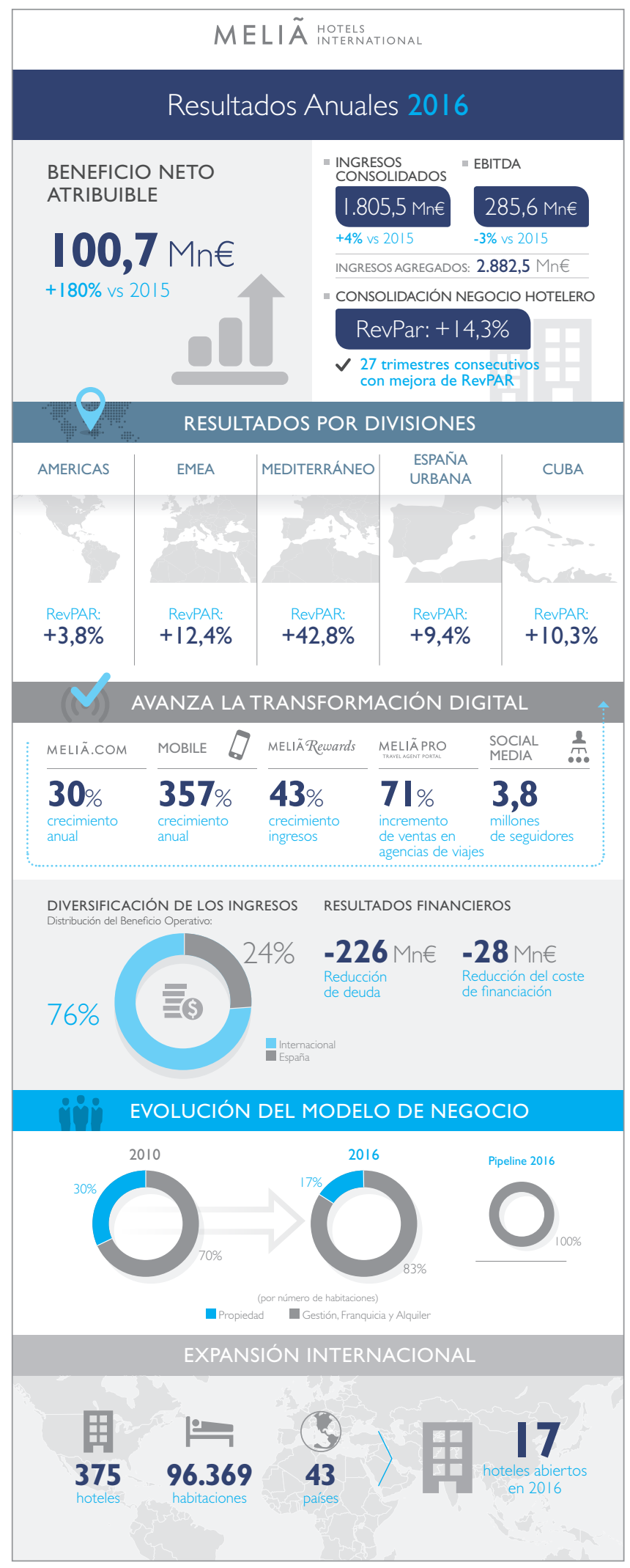

Figura 4. Storytelling de nivel 3 de Meliá. http://goo.gl/yLLpXD
Como caso de éxito del nivel 3, se encuentra Acciona por la variedad de aplicación de infografías, que oscilan desde los resultados financieros hasta sus procesos de negocio o sus diferentes proyectos.

En resultados financieros, Meliá es la empresa que mejor síntesis realiza de los datos de la memoria anual, de modo explicativo, a pesar de que su formato de archivo en PDF no facilite que se pueda compartir en algunas redes sociales. Tampoco incorpora un enlace a la información completa (figura 4).

En cuanto a la presentación de la historia de la empresa del nivel 4, Telefónica recoge los principales hechos relevantes no sólo para la compañía, sino también para la sociedad, en la línea del valor compartido que debe practicar cualquier empresa (Porter; Kramer, 2011). Estos acontecimientos son de interés para los usuarios, tienen una coherencia entre ellos y presentan interactividad a través del timeline (figura 5).

Sin embargo, la historia de Telefónica no se encuentra en la web corporativa, sino sólo en la de su Fundación.

\section{El storytelling digital corporativo está más implantado en el sector de teleco- municaciones del Dow Jones}

\section{Análisis del Dow Jones}

A pesar de que los resultados obtenidos son mejores que en el caso español, también son discretos. El 13,33\% de las empresas que cotizan en el Dow Jones disponen de una página de inicio basada en storytelling de nivel 1 , focalizado sobre la marca corporativa, servicios, productos, públicos y responsabilidad social, empleando también la animación parallax.

Es representativo el sector de telecomunicaciones, donde la mitad de las empresas cumple con los criterios mínimos establecidos para hablar de storytelling corporativo, como en los casos de Verizon o Intel.

El 13,33\% de las empresas norteamericanas presentan además storytelling al contar historias sobre sus públicos (nivel 2), ya sean clientes, empleados, proveedores... En Boeing se cuentan en primera persona historias de personas a las que un vuelo les cambió la vida y en Goldman Sachs sus narraciones tratan sobre cómo ha ayudado a los americanos a planear un futuro mejor. En Chevron sus historias se construyen sobre materias de actualidad. Como novedad, General Electric utiliza el vídeo 360 interactivo como soporte tecnológico, que lamentablemente prioriza sobre la propia narrativa. 


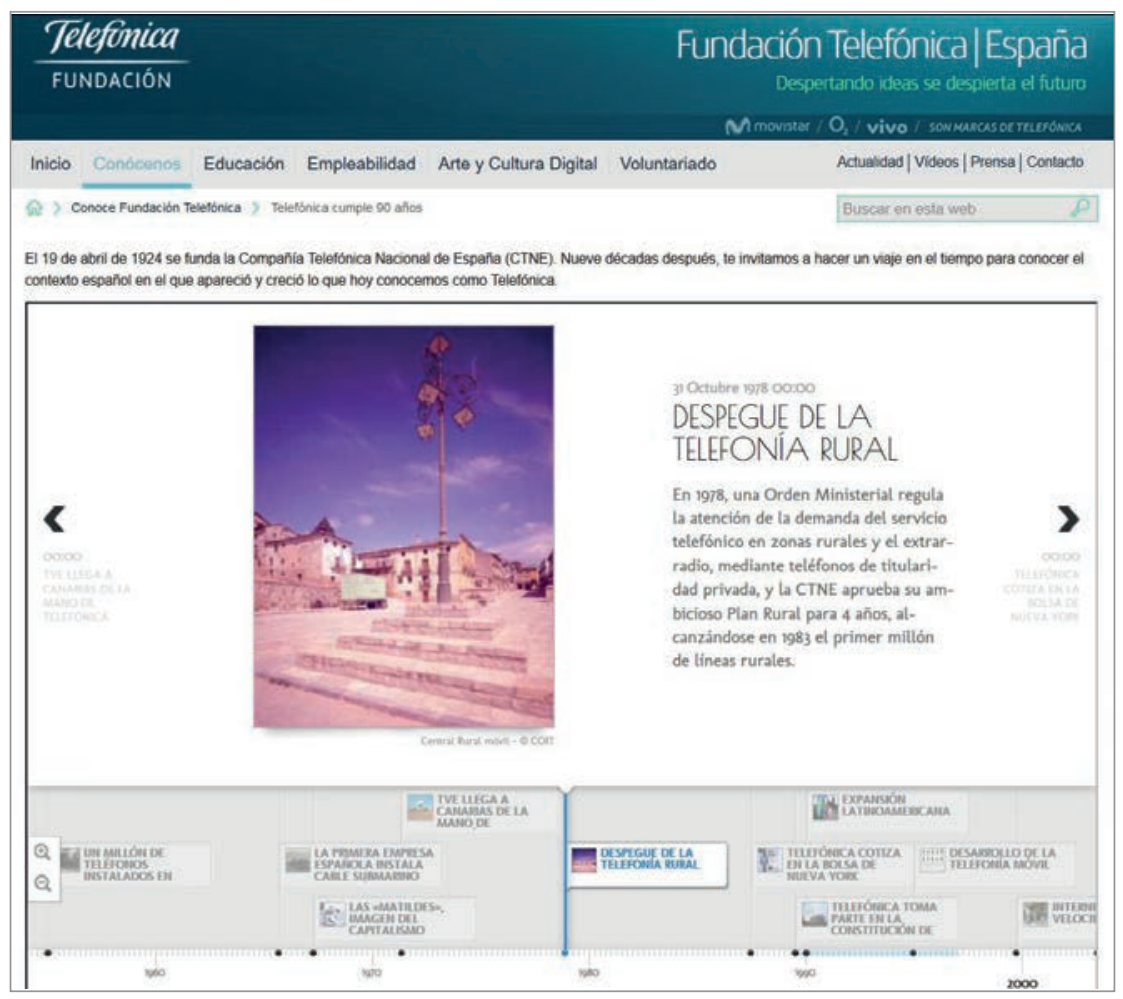

Figura 5. Storytelling de nivel 4 de Telefónica. $h t t p: / / g o o . g l / X Y 537 j$
Los niveles 3 y 4 están presentes en el $40 \%$ de las empresas, siendo petróleo y energía y tecnología y telecomunicaciones los sectores con menor presencia de estas dos categorías.

El nivel 3 está muy implantado en servicios de consumo (75\%), bienes de consumo (66\%) y materiales básicos, industria y construcción (60\%), siendo Coca-Cola la empresa que aglutina en una única infografía una narrativa relevante dirigida simultáneamente a los principales públicos de la compañía, lo que la diferencia del resto de empresas en esta categoría.

En el nivel 4, cuatro de los seis sectores registran un cumplimiento de entre el 40 y el $50 \%$ : bienes de consumo (50\%), servicios de consumo (50\%), servicios financieros e inmobiliarios (50\%) y materiales básicos, industria y construcción (40\%). El storytelling de nivel 4 en el timeline de las empresas norteamericanas es en la mayoría de los casos

Tabla 4. Presencia de categorías de storytelling por sectores del Dow Jones

\begin{tabular}{|c|c|c|c|c|}
\hline Categorías & Sectores & No empresas & $\%$ & $\%$ \\
\hline \multirow{6}{*}{ Nivel 1} & 1. Petróleo y energía & 0 & 0,00 & \multirow{6}{*}{13,33} \\
\hline & 2. Mat. básicos, industria y construcción & 0 & 0,00 & \\
\hline & 3. Bienes de consumo & 1 & 16,67 & \\
\hline & 4. Servicios de consumo & 0 & 0,00 & \\
\hline & 5. Servicios financieros e inmobiliarios & 0 & 0,00 & \\
\hline & 6. Tecnología y telecomunicaciones & 3 & 50,00 & \\
\hline \multirow{6}{*}{ Nivel 2} & 1. Petróleo y energía & 0 & 0,00 & \multirow{6}{*}{13,33} \\
\hline & 2. Mat. básicos, industria y construcción & 0 & 0,00 & \\
\hline & 3. Bienes de consumo & 0 & 0,00 & \\
\hline & 4. Servicios de consumo & 1 & 25,00 & \\
\hline & 5. Servicios financieros e inmobiliarios & 2 & 33,33 & \\
\hline & 6. Tecnología y telecomunicaciones & 1 & 16,67 & \\
\hline \multirow{6}{*}{ Nivel 3} & 1. Petróleo y energía & 1 & 33,33 & \multirow{6}{*}{40,00} \\
\hline & 2. Mat. básicos, industria y construcción & 3 & 60,00 & \\
\hline & 3. Bienes de consumo & 4 & 66,67 & \\
\hline & 4. Servicios de consumo & 3 & 75,00 & \\
\hline & 5. Servicios financieros e inmobiliarios & 1 & 16,67 & \\
\hline & 6. Tecnología y telecomunicaciones & 0 & 0,00 & \\
\hline \multirow{6}{*}{ Nivel 4} & 1. Petróleo y energía & 1 & 33,33 & \multirow{6}{*}{40,00} \\
\hline & 2. Mat. básicos, industria y construcción & 2 & 40,00 & \\
\hline & 3. Bienes de consumo & 3 & 50,00 & \\
\hline & 4. Servicios de consumo & 2 & 50,00 & \\
\hline & 5. Servicios financieros e inmobiliarios & 3 & 50,00 & \\
\hline & 6. Tecnología y telecomunicaciones & 1 & 16,67 & \\
\hline
\end{tabular}




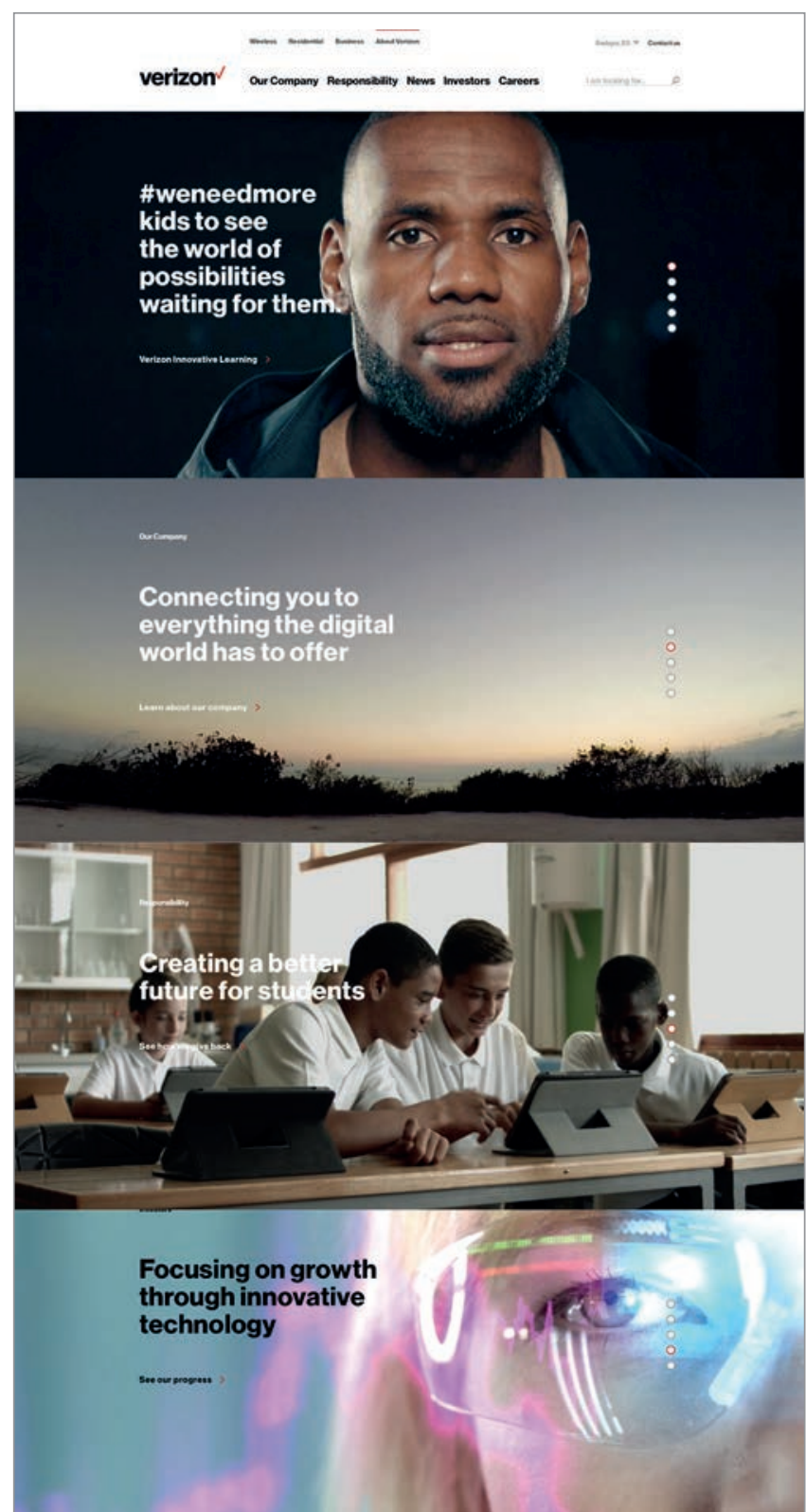

Figura 6. Storytelling de nivel 1 de Verizon. http://goo.gl/TSGByg

idéntica -exceptuando el contenido- al utilizado por Telefónica y que se ha presentado con anterioridad como caso de éxito. Un ejemplo es el de JPMorgan Chase \& Co (figura 8).

\section{Comparación IBEX 35 vs. Dow Jones}

La presencia de storytelling digital corporativo es superior en todos los niveles en las empresas del Dow Jones con respecto al IBEX 35. A pesar de que las mayores diferencias se aprecian en los niveles 1 y 2 , los niveles 3 y 4 presentan resultados similares, con presencia en torno al $40 \%$ de las empresas.

Este dato consolida el avance del storytelling en las webs corporativas, puesto que las únicas empresas estadounidenses que tienen implantados los niveles 1 y 2 provienen respectivamente de los sectores que destacan por una mayor presencia de los niveles 3 y 4 : servicios de consumo y

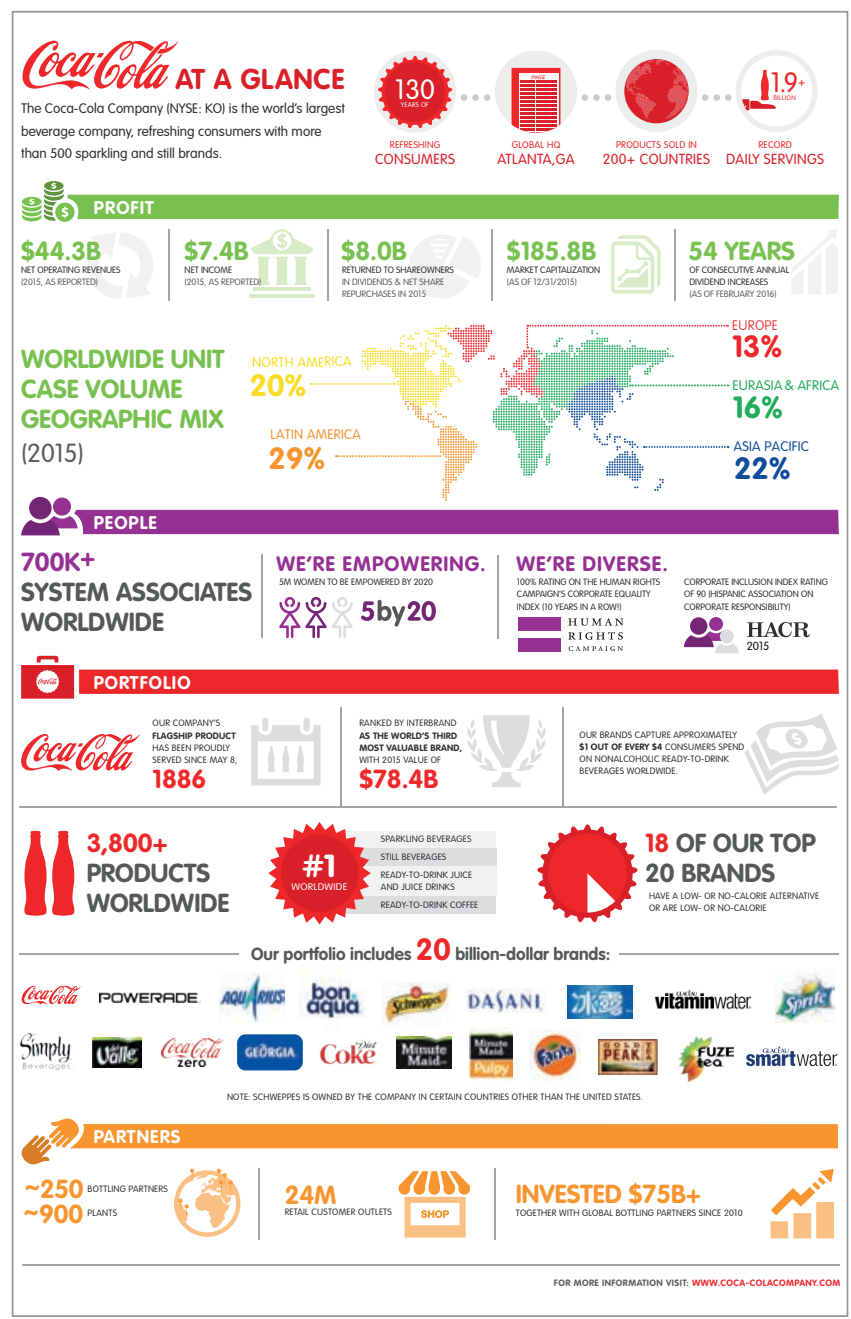

Figura 7. Storytelling de nivel 3 de Coca-Cola.

http://goo.gl/8QWpiy

bienes de consumo. Sin embargo, el sector tecnología y telecomunicaciones tiene la mayor implantación del nivel 1 , pero menor presencia en los niveles 3 y 4.

En el IBEX 35 sólo una empresa tiene implantado el nivel 2 y ninguna el nivel 1, mientras que la mayoría de los sectores presenta un grado de implantación del nivel 4 mayor que del nivel 3.

\section{Conclusiones}

La situación de las empresas estadounidenses en la implantación de storytelling digital corporativo es más avanzada que la de las españolas, aunque queda mucho camino por recorrer, sobre todo en los niveles 2 y 1 referidos a las stories y a la incorporación de storytelling en el index.

Aunque storytelling implica narrativa y contenidos, hay que considerar que determinadas tecnologías, como animaciones parallax, timelines, vídeos interactivos y 360 o o infografías, ayudan a mantener el interés del lector en las historias contadas.

Por tanto aportamos las siguientes recomendaciones sobre la integración del storytelling en webs corporativas, ejemplarizadas en algunos casos de éxito descritos: 
- Comenzar la estrategia de storytelling por el eslabón más sencillo, sustituyendo interminables textos por un timeline interactivo que recoja acontecimientos relevantes para la empresa, pero desde la perspectiva del interés de los públicos.

- Sintetizar la información financiera y el modelo de negocio en infografías que sean comprensibles, visuales y en formato imagen para que se compartan y fomenten la participación de los usuarios a la hora de interactuar con el contenido.

- Sustituir la tradicional estructura de la página de inicio basada en un resumen de las principales secciones de la web corporativa por historias que conecten emocionalmente con las personas, humanizando el quehacer de la empresa, su identidad y su narrativa.

Todo ello permitirá mejorar la reputación de la empresa a partir de la conexión emocional con sus públicos, siempre que el concepto de valor compartido guíe las actuaciones de la marca corporativa.

El storytelling digital corporativo implica nuevas oportunidades de especialización para los graduados en Comunicación, sustentadas en un cambio que las empresas deben afrontar para mejorar su credibilidad y vinculación emocional con los públicos.

Este trabajo inaugura una nueva línea de investigación dentro del storytelling. En próximas investigaciones se debe determinar en qué grado éste mejora la vinculación emocional entre empresa y público en el contexto de su estrategia digital corporativa.

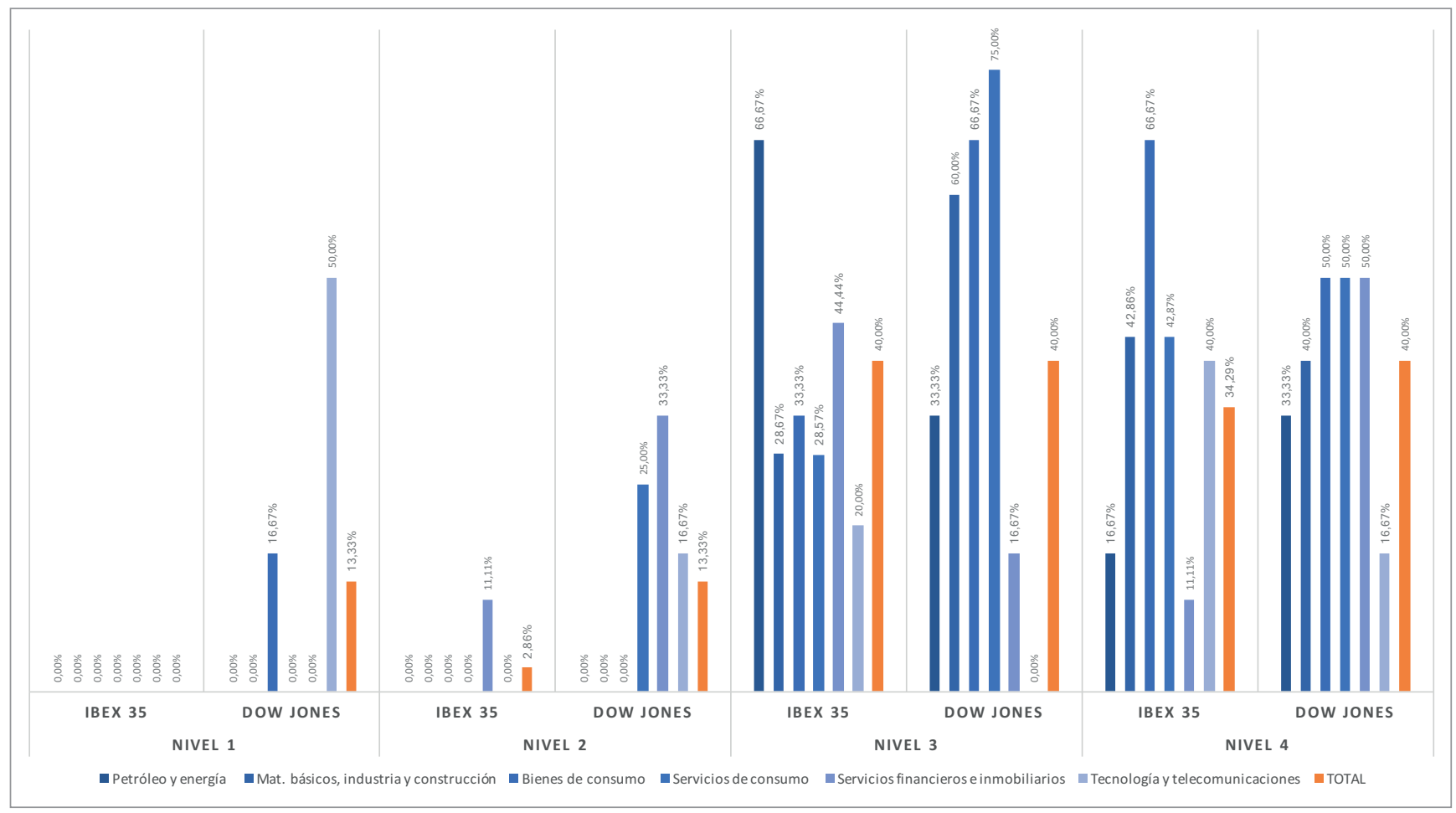

Figura 9. Presencia de storytelling digital corporativo en las empresas del IBEX 35 con respecto a las del Dow Jones 


\section{Referencias}

Adecec (2015). Guía storytelling y branded content. http://www.adecec.com/pdf/la-guia-de-storytelling-ybranded-content-de-adecec.pdf

Alexander, Bryan; Levine, Alan (2008). "Web 2.0 storytelling. Emergence of a new genre". Educause review, v. 43, n. 6, pp. 40-56.

https://er.educause.edu/articles/2008/10/web-20storytelling-emergence-of-a-new-genre

Barciela, Fernando (2013). "El último desafío, el 'brand journalism'". Cuadernos de periodistas, n. 26, pp. 125-136. http://www.apmadrid.es/wp-content/uploads/2013/06/125-136. $p d f$

Benavides, Juan (2013). "Un nuevo enfoque para la comprensión de la marca y la comunicación de la empresa". En: De-Salas-Nestares, María-Isabel; Mira-Pastor, Enric (2013). Prospectivas y tendencias para la comunicación en el siglo XXI. Valencia: CEU Universidad Cardenal Herrera, pp. 515543. ISBN: 9788415949176

Bull, Andy (2013). Brand journalism. New York: Routledge. ISBN: 9780415638104

Cairo, Alberto (2008). Infografía 2.0: visualización interactiva de información en prensa. Madrid: Alamut. ISBN: 978 8498890105

Cole, James; Greer, Jennifer (2013). “Audience response to brand journalism. The effect of frame, source, and involvement". Journalism \& mass communication quarterly, v. 90, n. 4, pp. 673-690.

https://goo.gl/NXbR5X

https://doi.org/10.1177/1077699013503160

Colle, Raymond (2004). "Infografía: tipologías". Revista latina de comunicación social, v. 7, n. 58, pp. 669-686.

https://www.ull.es/publicaciones/latina/latina_art660.pdf

Costa-Sánchez, Carmen (2014). "Storytelling y audiovisualización de la comunicación corporativa: las claves de la campaña 'Gracias por elegirnos' (Balay)”. Organicom, v. 11, n. 20, pp. 163-176.

http://revistaorganicom.org.br/sistema/index.php/organicom/ article/view/698

Durántez-Stolle, Patricia (2017). “Interacción y participación en salas de prensa virtuales de las empresas del IBEX 35". El profesional de la información, v. 26, n. 2, pp. 228-237. https://doi.org/10.3145/epi.2017.mar.09

Fog, Klaus; Budtz, Christian; Yakaboylu, Baris (2005). Storytelling. Branding in practice. Berlin: Springer. ISBN: 978 3540883487

Fundación Compromiso y Transparencia (2013). Los 10 mayores errores sobre transparencia, supervisión y buen gobierno 2011-2012.

http://www.compromisoempresarial.com/wp-content/ uploads/Los-10errores.pdf

González-Ortín, Mónica (coord.) (2014). Nuevos retos y oportunidades de los departamentos de comunicación en España. Madrid: Axicom-PRNoticias.com. $h t t p: / / g o o . g l / q A b B Z I$

Herrero-Curiel, Eva (2015). "Claves en la era de la pospublicidad. El storytelling y la narración transmediáticas". Revista Telos. Cuadernos de comunicación e innovación, n. 102, pp. 1-5. http://goo.gl/xYjaVC

Johndrow, Anthony (2010). "Making money in the reputation economy". Forbes, 27 agosto.

http://goo.gl/qwDnUO

Lugmayr, Artur; Sutinen, Erkki; Suhonen, Jarkko; Islas-Sedano, Carolina; Hlavacs, Helmut; Suero-Montero, Calkin (2017). "Serious storytelling - a first definition and review". Multimedia tools and applications, v. 76, n. 14, pp. 1-27.

https://goo.gl/wsHwrJ

https://doi.org/10.1007/s11042-016-3865-5

Marketingdirecto.com (2015). "Heineken España lanza su nueva web corporativa basada en el storytelling". Marketingdirecto.com, 5 febrero.

https://goo.gl/P6poYW

Martín-González, José-Antonio (2009). "La eficacia del storytelling". MK Marketing+Ventas, n. 251, pp. 8-16. http://pdfs.wke.es/9/8/2/2/pd0000049822.pdf

Martín-Requero, María-Isabel; Alvarado-López, María-Cruz (coord.) (2007). Nuevas tendencias en la publicidad del siglo XXI. Sevilla: Comunicación Social. ISBN: 9788496082519

Nakevska, Marij et al. (2017). "Interactive storytelling in a mixed reality environment: The effects of interactivity on user experiences". En: Pisan, Yusuf; Sgouros, Nikitas-Marinos; Marsh, Tim (eds.). Entertainment computing - ICEC 2014. Berlin: Springer, pp. 52-59. ISBN: 9783662452110 https://doi.org/10.1007/978-3-662-45212-7_7

O'Reilly, Tim (2005). "What is web 2.0". O'Reilly.com, 30 Sept. http://www.oreilly.com/pub/a/web2/archive/what-isweb-20.html

Pereira-Lima, Edvaldo (2014). "Storytelling em plataforma impressa e digital: contribuição potencial do jornalismo literário". Organicom, v. 11, n. 20, pp. 119-127.

http://www.revistaorganicom.org.br/sistema/index.php/ organicom/article/view/692

Pérez-Montoro, Mario (2014). “Políticas universitarias de difusión de la información a través de la propia web institucional". El profesional de la información, v. 23, n. 2, pp. 190-194. https://doi.org/10.3145/epi.2014.mar.12

Pineda-Martínez, Paula (2013). "Relaciones públicas online de excelencia: las empresas energéticas del IBEX 35 ante el reto de comunicar su conducta responsable". Revista internacional de relaciones públicas, v. 3, n. 5, pp. 189-208. https://doi.org/10.5783/RIRP-5-2013-11-189-208

Porter, Michael E.; Kramer, Mark R. (2011). "The big idea: Creating shared value". Harvard business review, v. 89, n. 1/2, pp. 62-77. https://goo.gl/1qTCh4

Quivy, Raymond; Van-Campenhoudt, Luc (2008). Manual de investigação em ciências sociais (4⿳亠口冋 ed.). Lisboa: Gradiva. ISBN: 9789726622758 
Roberts, Kevin (2005). Lovemarks: el futuro más allá de las marcas. Madrid: Empresa activa. ISBN: 9788495787804 https://blogdemarqueting.files.wordpress.com/2013/12/ lovemarks-kevin-roberts.pdf

Salmon, Christian (2008). Storytelling. La máquina de fabricar historias y formatear las mentes. Barcelona: Península. ISBN: 9788483078358

Sánchez-Revilla, Miguel-Ángel (2017). Estudio Infoadex de la inversión publicitaria en España 2016. Madrid: Infoadex. ISBN: 9788493927691

https://goo.gl/kUn52p

Tapia-Frade, Alejandro; Caerlos-Mateo, Raquel (2014). “La exposición de información corporativa en página web: el caso de las empresas del IBEX 35". Sphera publica, v. 2, n. 14, pp. 155-175.

http://sphera.ucam.edu/index.php/sphera-01/article/view/233
Túñez-López, Miguel; Nogueira, Ana-Gabriela (2017). “Infographics as a mnemonic structure: Analysis of the informative and identity components of infographic online compositions in Iberic newspapers". Communication \& society, v. 30, n. 1, pp. 147-164.

https://doi.org/10.15581/003.30.1.147-164

Uspto (1903). Parallax stereogram and process of making same. N. 725,567.

http://goo.gl/4wBa1p

UXPin (2015). Web design book of trends 2015-2016. Gdynia: UXPin Inc.

http://goo.gl/1Yph9i

Valero-Sancho, José-Luis (2008). "La infografía digital en el ciberperiodismo". Revista latina de comunicación social, n. 63, pp. 492-504.

https://doi.org/10.4185/RLCS-63-2008-799-492-504

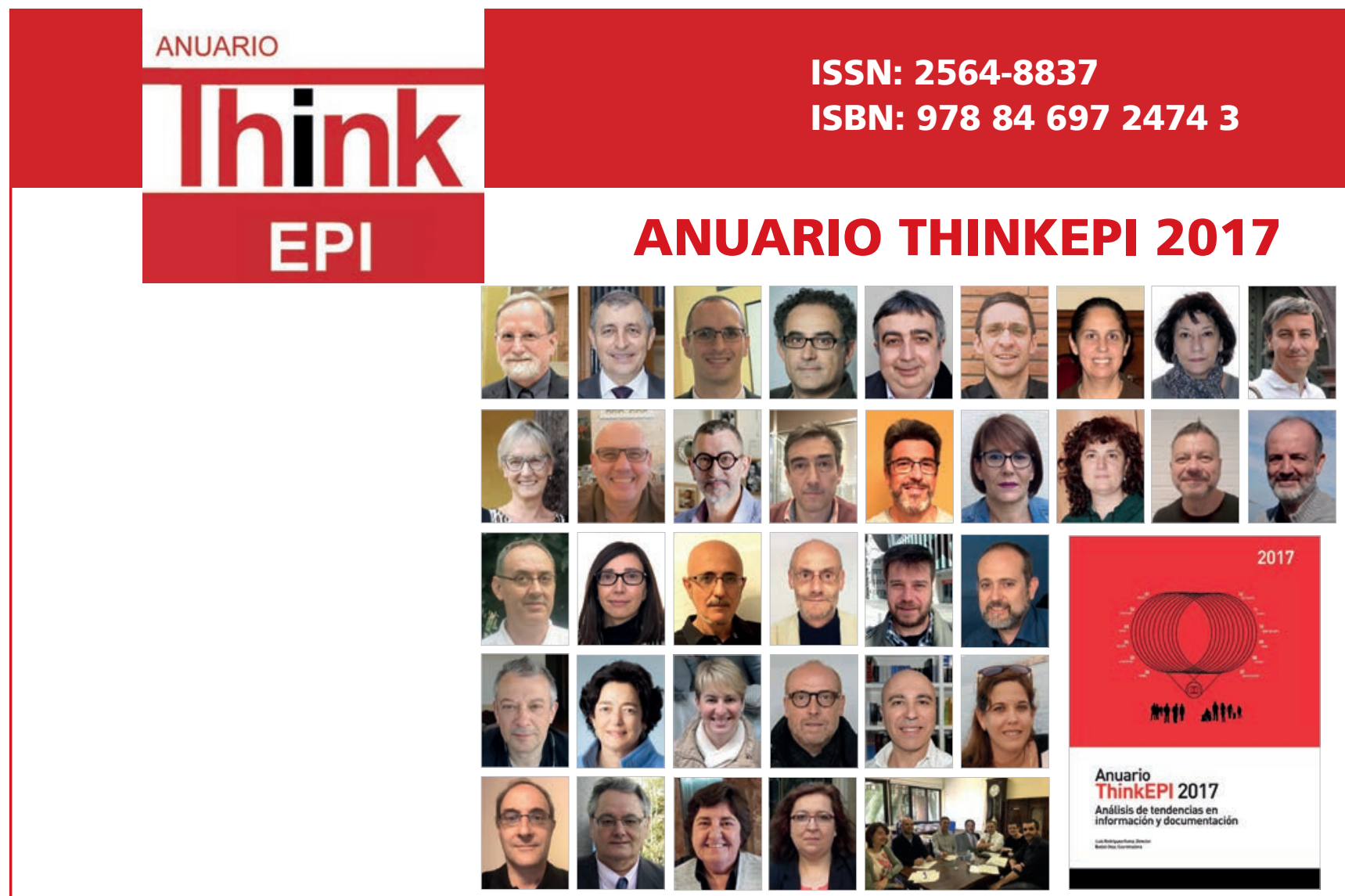

\section{PRECIOS ANUARIO THINKEPI}

Suscripción online (2007-2017)

Instituciones ......................................... $85 €$

Individuos (particulares) .................. $51 €$

Números sueltos

Instituciones

Anuario ThinkEPI 2017 (pdf) .............40 $40 €$

Anuario de ańos anteriores .............. $20 €$

Individuos (particulares)

Anuario ThinkEPI 2017 (pdf)

Anuario de ańos anteriores

$20 €$
Es posible el acceso mediante suscripción a todos los Anuarios ThinkEPI publicados hasta el momento desde el Recyt de la Fecyt http://recyt.fecyt.es/index.php/ThinkEPI

\section{Más información: Isabel Olea epi.iolea@gmail.com}

